Article

\title{
Simplified Modelling of Inclined Turbulent Dense Jets
}

\author{
Ilias G. Papakonstantis *(D) and George C. Christodoulou
}

Laboratory of Applied Hydraulics, Department of Water Resources and Environmental Engineering, School of Civil Engineering, National Technical University of Athens, 5 Heroon Polytechniou Street, 15780 Zografou, Greece; christod@hydro.ntua.gr

* Correspondence: ipapak@mail.ntua.gr; Tel.: +30-2107722876

Received: 12 October 2020; Accepted: 9 November 2020; Published: 10 November 2020

\begin{abstract}
An analytical approximation to the entire centerline trajectory of inclined round dense jets in dimensionless form is proposed, in terms of a fourth degree polynomial. The coefficients of the polynomial for a certain inclination angle can be easily obtained if the position of the maximum height and the return point are known. Experimental data of the authors are used to determine these coefficients for six inclination angles between $35^{\circ}$ and $75^{\circ}$. The resulting trajectories are then compared to data of other investigators and found to be in good agreement. The variation of the polynomial coefficients with inclination angle is also studied. The proposed analytical expression allows for a straightforward computation of the trajectory length for any inclination angle in the range studied. It is found that the longest trajectory occurs for the $60^{\circ}$ angle. The relation between the computed length and the measured minimum (centerline) dilutions at the location of maximum height and at the return point is examined. Finally, the laws governing the variation of the minimum dilution with the axial distance from the source are explored and similarities with the laws of simple jets and plumes are discussed.
\end{abstract}

Keywords: inclined dense jets; negatively buoyant jets; turbulent jets; jet trajectory; dilution; desalination brines

\section{Introduction}

Desalination plants are rapidly increasing worldwide in coastal areas with water scarcity due to population growth and consequent freshwater needs. Typically, brines from desalination plants are discharged back into the sea by means of single or multi-port outfalls. Such effluents may become a major source of marine pollution, particularly in the benthic zone, as they contain high salt concentrations and traces of chemicals, e.g., chlorine, coagulants, antifoaming additives, etc. [1]. It is therefore desirable to achieve a high dilution of the brine effluent, prior to its impact on the benthic zone. To this end, normally the discharge is directed upwards at an angle, leading to the formation of inclined dense jets, i.e., jets with negative buoyancy. These typically consist of an upward and a downward branch: the former is driven by the initial vertical component of the jet momentum and extends up to the point where this vanishes (maximum height of rise); the downward branch then follows, ending at the bottom of the ambient water body.

The geometry, dilution and other features of the flow field within an inclined round dense jet have been studied experimentally by numerous investigators. Zeitoun et al. [2] presented results for the terminal height and the return distance for discharge angles $30^{\circ}, 45^{\circ}$ and $60^{\circ}$ and they concluded that the $60^{\circ}$ angle yields the longest trajectory and consequently the highest dilution at the return region. Subsequently, Roberts and Toms [3] and Roberts et al. [4] conducted experiments for the $60^{\circ}$ inclined dense jet, whereas Cipollina et al. [5] studied jets inclined at $30^{\circ}, 45^{\circ}$ and $60^{\circ}$. Nemlioglu and Roberts [6] 
used laser induced fluorescence (LIF) to obtain results for the main geometrical characteristics and dilution for discharge angles between $15^{\circ}$ and $90^{\circ}$. Kikkert [7] and Kikkert et al. [8] employed light attenuation (LA) and LIF techniques to study the trajectory characteristics, concentration distributions and dilution of dense jets discharged at angles between $0^{\circ}$ and $75^{\circ}$. Ferrari and Querzoli [9] also conducted LIF experiments for angles between $45^{\circ}$ and $90^{\circ}$, focusing on the re-entrainment occurring for angles exceeding $75^{\circ}$. Shao and Law [10] studied jets inclined at $30^{\circ}$ and $45^{\circ}$ using particle image velocimetry (PIV) and LIF techniques. Papakonstantis et al. [11] presented experimental results based on flow visualization for the geometrical characteristics of the jet upper boundary for six discharge angles between $45^{\circ}$ and $90^{\circ}$, whereas Papakonstantis et al. [12] reported results for concentration distributions, dilution and geometrical characteristics of the jet centerline obtained from concentration measurements in dense jets inclined at $45^{\circ}, 60^{\circ}$ and $75^{\circ}$. Lai and Lee [13] conducted experiments for six discharge angles between $15^{\circ}$ and $60^{\circ}$ using LIF and PIV and reported results for geometrical characteristics, concentration distributions, dilution and velocity. Oliver [14] and Oliver et al. [15] employed the LIF technique to study dense jets inclined at several angles between $15^{\circ}$ and $75^{\circ}$ and obtained results for jet trajectory, concentration distributions and dilution. Abessi and Roberts [16] conducted LIF experiments for dense jets discharged at angles between $15^{\circ}$ and $85^{\circ}$ at $5^{\circ}$ intervals and obtained geometrical characteristics and dilution data for the jet flow and the dense layer spreading on the bottom. Results for the angles $30^{\circ}, 45^{\circ}$ and $60^{\circ}$ have also been reported by Abessi and Roberts [17]. Data regarding the velocity field and the jet geometrical characteristics were reported by Crowe et al. [18] for discharge angles between $15^{\circ}$ and $75^{\circ}$. Papakonstantis and Tsatsara [19] reported results from flow visualization for trajectory characteristics of the upper jet boundary for five discharge angles between $15^{\circ}$ and $70^{\circ}$, whereas Papakonstantis and Tsatsara [20] studied centerline trajectory characteristics and dilution of dense jets discharged at angles of $35^{\circ}, 50^{\circ}$ and $70^{\circ}$. Several experimental studies have revealed the complexity of the flow field. In particular, at the region of the maximum height, buoyancy-driven instabilities develop in the inner side of the jet leading to the separation of fluid parcels from the main flow, e.g., $[8,11]$.

Besides the experimental studies, several works have been presented for modelling inclined round dense jets including analytical approaches, integral models and computational fluid dynamics (CFD) tools. More specifically, Vafeiadou et al. [21] conducted numerical simulations of inclined dense jets using CFX software. Oliver et al. [22] employed the $\mathrm{k}-\varepsilon$ turbulence model and found that the predictions for bulk flow parameters are not superior to those provided by the analytical solutions of Kikkert et al. [8]. Gildeh et al. [23,24] studied the $30^{\circ}$ and $45^{\circ}$ inclined dense jets by means of CFD simulations, whereas Zhang et al. $[25,26]$ employed the large eddy simulation (LES) approach for the $45^{\circ}$ and $60^{\circ}$ jets. As far as integral modeling is concerned, Papanicolaou et al. [27] proposed a reduced value of the jet entrainment coefficient $\alpha j$ whereas Lai and Lee [13] allowed negative values of the local Froude number yielding a reduction in the entrainment coefficient below the normal value of $\alpha j$ in the rising branch of the flow. Yannopoulos and Bloutsos [28] introduced the escaping mass approach (EMA) for explicitly modeling the loss of mass from the concave side of the jet due to vertical escape of fluid parcels. Oliver et al. [29] proposed a reduced buoyancy flux model, in which the buoyancy flux is allowed to decrease up to the maximum height and is kept constant beyond that point. The application of commercial codes, such as Corjet and Visjet, has also been attempted, but with limited success [30]. Nikiforakis et al. [31] proposed a modification of Corjet to take explicitly into account the detrainment of fluid. Christodoulou and Papakonstantis [32] suggested a parabolic approximation of the dimensionless jet trajectory and they determined the coefficients of the parabola based on three boundary conditions. The agreement with experimental results was reasonably good, although the actual trajectory is not parabolic but rather skewed, e.g., [14].

The objective of this paper is to provide simplified modelling of inclined round dense jets in the form of simple analytical expressions for determining with sufficient accuracy the centerline trajectory as well as the minimum dilution at key positions. Such modelling is not expected to describe details of the complex flow field of negatively buoyant jets, but it can be an efficient tool for design 
purposes, providing fast and easy solutions, without resort to experiments or elaborate numerical models. The study focuses on the most common and practically important range of angles, $35^{\circ}$ to $75^{\circ}$.

\section{Theoretical Considerations}

A schematic and the main notation of an inclined dense jet is shown in Figure 1. Fluid of density $\rho$, discharges with velocity $U_{o}$ from a pipe of diameter $D$ at an angle $\theta$, into a stagnant homogeneous ambient fluid of slightly smaller density $\rho_{\alpha}$ such that $\left(\rho_{o}-\rho_{\alpha}\right) / \rho_{\alpha}<<1$, i.e., the Boussinesq approximation is valid. Geometrical characteristics of the jet centerline are measured with respect to a coordinate system located at the jet exit pipe. The maximum centerline height $Z_{c}$ is observed at a horizontal distance $X_{Z}$ from the source, while $X_{i c}$ is the horizontal distance from the source to the centerline return point at the level of the jet exit pipe. Minimum (centerline) dilutions at the maximum height and the return point are denoted as $S_{t}$ and $S_{i}$, respectively.

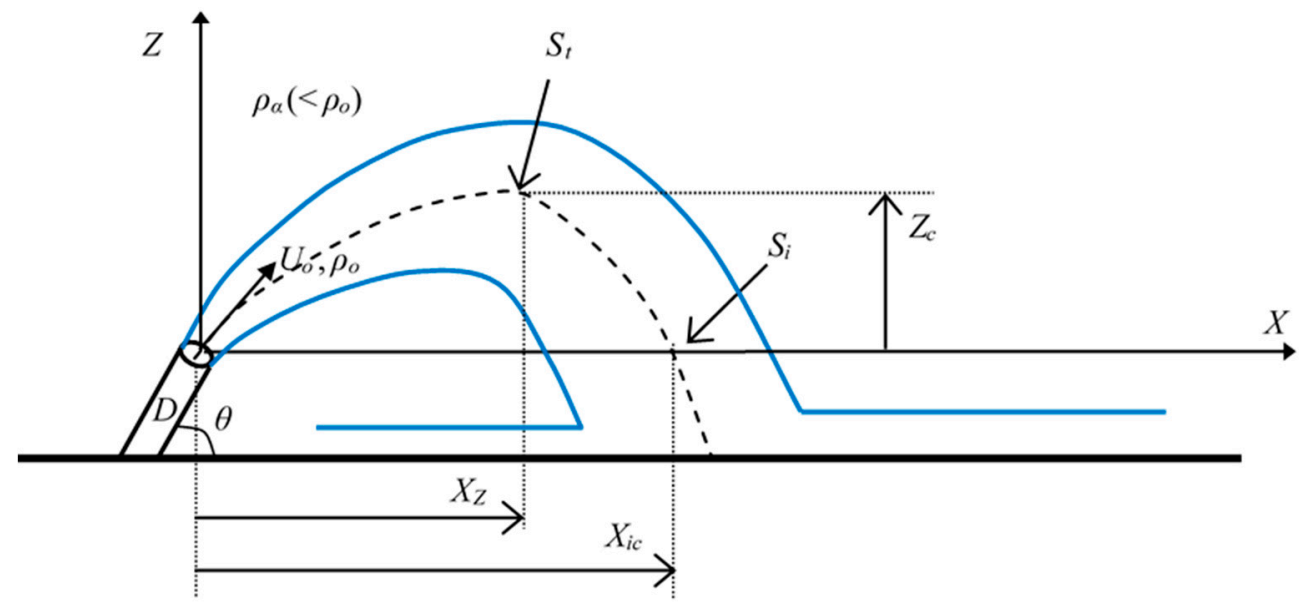

Figure 1. Schematic and main notation of an inclined dense jet.

It has been well established through dimensional analysis $[4,11,12]$ that for turbulent jets at relatively large densimetric Froude numbers, any geometrical characteristic $Y$ of the jet can be expressed as

$$
\frac{Y}{D F_{o}}=C(\theta)
$$

where $F_{0}$ is the densimetric Froude number

$$
F_{o}=\frac{U_{o}}{\sqrt{g_{o}^{\prime} D}}
$$

with $g_{o}^{\prime}=g \frac{\rho-\rho_{\alpha}}{\rho_{\alpha}}$ being the apparent acceleration of gravity at the source.

Equation (1) implies that the dimensionless coordinates of the jet trajectory depend solely on the discharge angle, therefore there is a single dimensionless trajectory for a given discharge angle. As a consequence, the maximum centerline height $Z_{c}$ and the respective horizontal distance $X_{Z}$ as well as the distance to the return point $X_{i c}$ can be written in dimensionless form as $[19,20]$ :

$$
\begin{aligned}
\frac{Z_{c}}{D F_{o}} & =C_{1}(\theta) \\
\frac{X_{Z}}{D F_{o}} & =C_{2}(\theta) \\
\frac{X_{i c}}{D F_{o}} & =C_{3}(\theta)
\end{aligned}
$$


Similarly, the dilution at any point of the dimensionless trajectory can be expressed as

$$
\frac{S}{F_{0}}=C_{4}(\theta)
$$

The coefficients $C_{1}, C_{2}, C_{3}, C_{4}$ vary with the discharge angle.

The validity of the above equations has been verified with extensive experimental research and the coefficients of those equations were determined by several investigators, as discussed in the Introduction.

\section{Simplified Analytical Model}

As discussed above, there is a single dimensionless trajectory for a given discharge angle. Christodoulou and Papakonstantis [32] proposed a parabolic approximation for the centerline trajectory and they determined the coefficients of the parabola by employing three boundary conditions, namely the origin $(0,0)$, the maximum centerline height $\left(X_{Z}, Z_{c}\right)$ and the return point $\left(X_{i c}, 0\right)$ obtained from available experimental data at specific inclination angles. However, experimental evidence shows that in fact the trajectory is not symmetric but rather skewed. Herein, a better approximation is sought by considering two additional boundary conditions, i.e., the known inclination at the origin and the condition of zero derivative at the point of maximum centerline height. Thus, the trajectory may be described by a fourth degree polynomial, as follows:

$$
Z^{*}=\alpha_{0}+\alpha_{1} X^{*}+\alpha_{2} X^{* 2}+\alpha_{3} X^{* 3}+\alpha_{4} X^{* 4}
$$

where $X^{*}$ and $Z^{*}$ denote the dimensionless variables $X /\left(D F_{0}\right)$ and $Z /\left(D F_{0}\right)$, respectively. Differentiating Equation (7) yields:

$$
\frac{d Z^{*}}{d x}=\alpha_{1}+2 \alpha_{2} X^{*}+3 \alpha_{3} X^{* 2}+4 \alpha_{4} X^{* 3}
$$

The five boundary conditions mentioned above lead to the following equations:

$$
\begin{gathered}
Z^{*}(0)=0 \Rightarrow a_{0}=0 \\
\frac{d Z^{*}}{d x}(0)=\tan (\theta) \Rightarrow \alpha_{1}=\tan (\theta) \\
Z^{*}\left(X_{Z}^{*}\right)=Z_{c}^{*} \Rightarrow \tan (\theta) X_{Z}^{*}+\alpha_{2} X_{Z}^{* 2}+\alpha_{3} X_{Z}^{* 3}+\alpha_{4} X_{Z}^{* 4}=Z_{c}^{*} \\
\frac{d Z^{*}}{d x}\left(X_{Z}^{*}\right)=0 \Rightarrow \tan (\theta)+2 \alpha_{2} X_{Z}^{*}+3 \alpha_{3} X_{Z}^{* 2}+4 \alpha_{4} X_{Z}^{* 3}=0 \\
Z^{*}\left(X_{i c}^{*}\right)=0 \Rightarrow \tan (\theta) X_{i c}^{*}+\alpha_{2} X_{i c}^{* 2}+\alpha_{3} X_{i c}^{* 3}+\alpha_{4} X_{i c}^{* 4}=0
\end{gathered}
$$

If for a certain inclination angle $\theta$ the values of $X_{Z}^{*}, Z_{c}^{*}, X_{i c}^{*}$ are known from experiments, Equations (11)-(13) form a linear system allowing a straightforward determination of the coefficients $a_{2}, a_{3}, a_{4}$. To implement the above, the experimental values of Papakonstantis et al. [11,12] and Papakonstantis and Tsatsara $[19,20]$ will be used, as they were all obtained in the same experimental apparatus and cover a wide range of discharge angles from $35^{\circ}$ to $75^{\circ}$. The experimental data used are summarized in Table 1 . The resulting trajectories are subsequently compared to the experimental results of other studies to test the degree of their validity. 
Table 1. Experimental data used to obtain the coefficients $\alpha_{2}, \alpha_{3}, \alpha_{4}$.

\begin{tabular}{cccccc}
\hline $\boldsymbol{\theta}$ & $\boldsymbol{Z}_{\boldsymbol{c}} / \boldsymbol{D} \boldsymbol{F}_{\boldsymbol{o}}$ & $\boldsymbol{X}_{\boldsymbol{Z}} / \boldsymbol{D} \boldsymbol{F}_{\boldsymbol{o}}$ & $\boldsymbol{X}_{\boldsymbol{i c}} / \boldsymbol{D} \boldsymbol{F}_{\boldsymbol{o}}$ & $\boldsymbol{S}_{t} / \boldsymbol{F}_{\boldsymbol{o}}$ & $\boldsymbol{S}_{\boldsymbol{i}} / \boldsymbol{F}_{\boldsymbol{o}}$ \\
\hline $35^{\circ}$ & 0.79 & 1.90 & 3.23 & 0.45 & 1.20 \\
$45^{\circ}$ & 1.17 & 2.03 & 3.16 & 0.52 & 1.55 \\
$50^{\circ}$ & 1.28 & 1.94 & 3.00 & 0.57 & 1.69 \\
$60^{\circ}$ & 1.68 & 1.83 & 2.75 & 0.56 & 1.68 \\
$70^{\circ}$ & 1.81 & 1.22 & 1.94 & 0.47 & 1.76 \\
$75^{\circ}$ & 1.93 & 1.15 & 1.80 & 0.51 & 1.67 \\
\hline
\end{tabular}

\section{Results and Discussion}

\subsection{Centerline Trajectory}

The linear system of Equations (11)-(13) was solved for each angle using Cramer's rule in Microsoft Excel. The obtained values of the coefficients are given in Table 2. The resulting trajectories for the angles $\theta=35^{\circ}, 45^{\circ}, 50^{\circ}, 60^{\circ}, 70^{\circ}, 75^{\circ}$ are shown in Figure 2.

Table 2. Coefficients of Equation (7) for various discharge angles.

\begin{tabular}{ccccccc}
\hline $\boldsymbol{\theta}$ & $\mathbf{3 5}^{\circ}$ & $\mathbf{4 5}^{\circ}$ & $\mathbf{5 0}^{\circ}$ & $\mathbf{6 0}^{\circ}$ & $\mathbf{7 0}^{\circ}$ & $\mathbf{7 5}^{\circ}$ \\
\hline$a_{0}$ & 0.0000 & 0.0000 & 0.0000 & 0.0000 & 0.0000 & 0.0000 \\
$a_{1}$ & 0.7002 & 1.0000 & 1.1918 & 1.7321 & 2.7475 & 3.7321 \\
$a_{2}$ & -0.1187 & -0.3461 & -0.4999 & -1.0070 & -1.6425 & -3.5900 \\
$a_{3}$ & 0.0038 & 0.1724 & 0.2666 & 0.6454 & 1.1419 & 2.8536 \\
$a_{4}$ & -0.0106 & -0.0516 & -0.0775 & -0.1848 & -0.5285 & -1.1172 \\
\hline
\end{tabular}

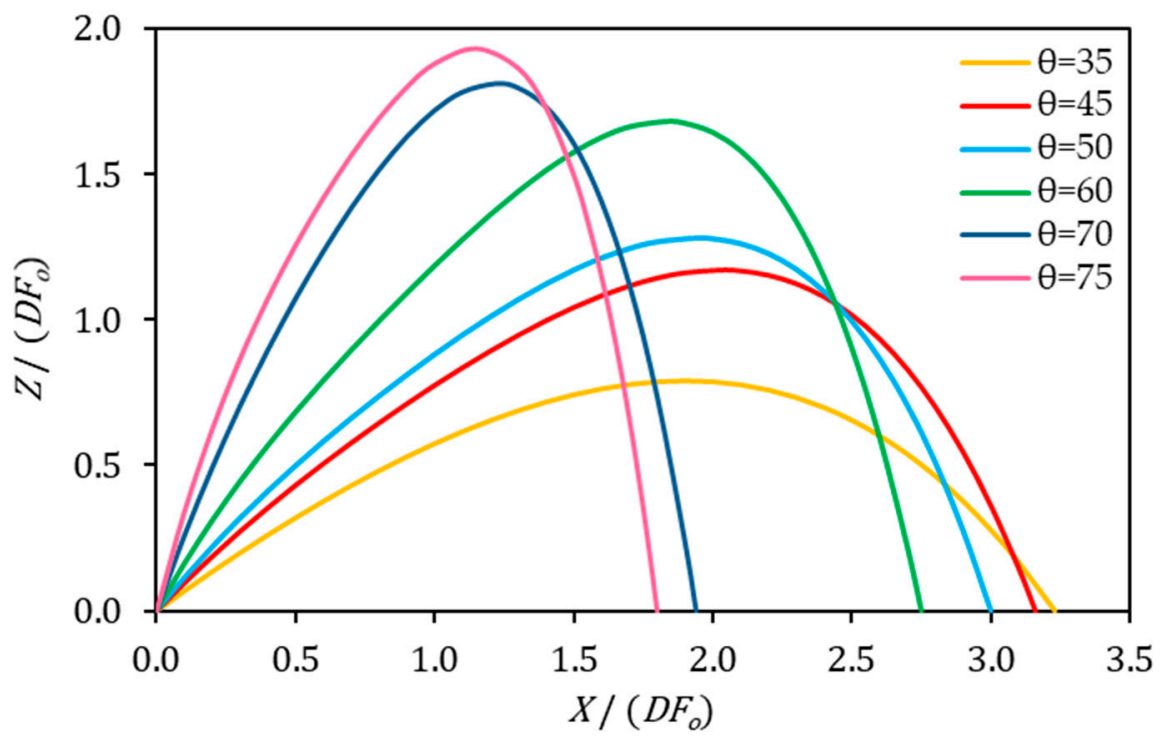

Figure 2. Dimensionless trajectories according to the fourth degree polynomial (Equation (7)) with coefficients as in Table 1 for discharge angles between $35^{\circ}$ and $75^{\circ}$.

To test the validity of the trajectories predicted by Equation (7), they are compared to detailed experimental data of other investigators for two discharge angles, namely $45^{\circ}$ and $60^{\circ}$. Selected data from those reported by Kikkert [7], Lai and Lee [13] and Oliver [14] are plotted in Figure 3. It can be seen that the predicted trajectories are in satisfactory agreement with the experimental data. In addition, as the literature includes a lot of data for the maximum centerline height and the return point for several inclination angles, a comparison is shown in Figure 4. Again, the location of these important points is generally well predicted by the proposed trajectories. 


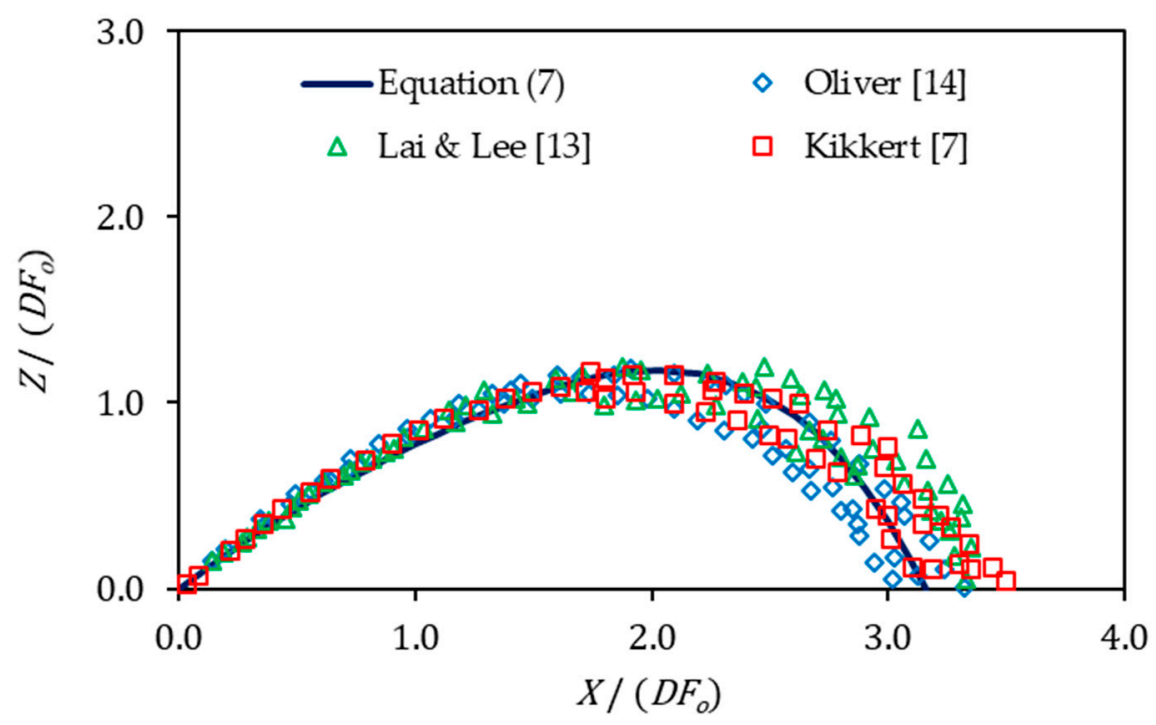

(a)

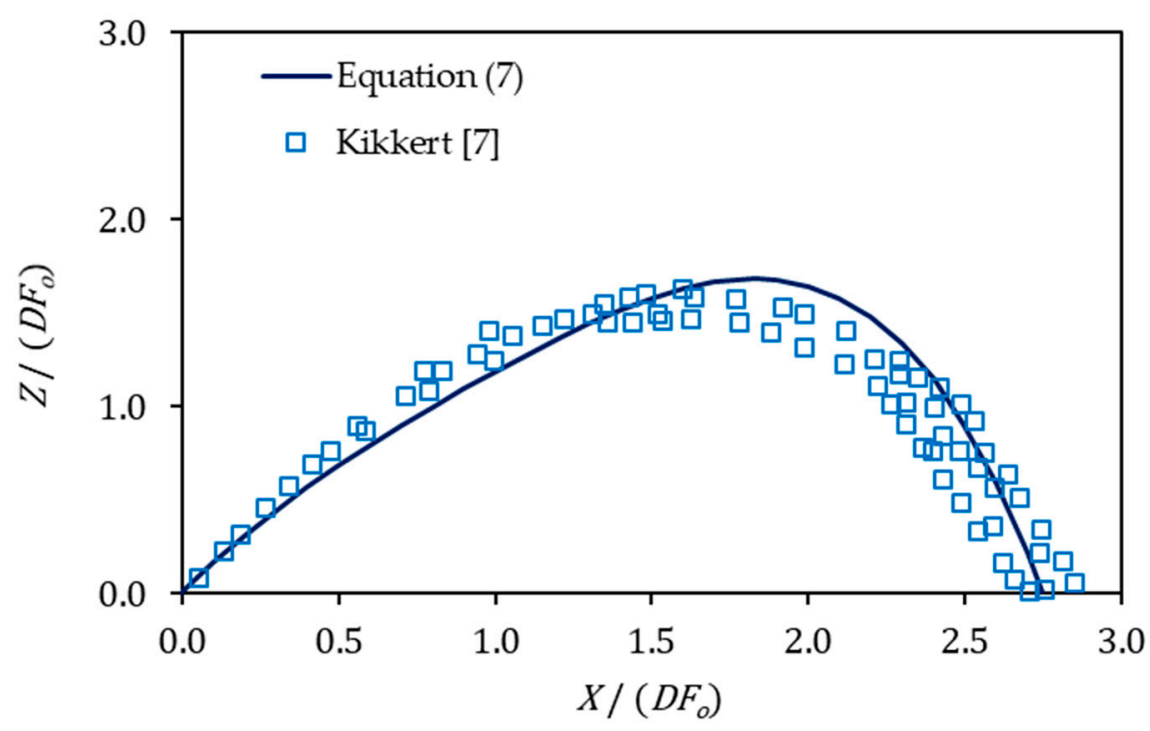

(b)

Figure 3. Comparison of the predicted centerline trajectories for (a) $\theta=45^{\circ}$, (b) $\theta=60^{\circ}$ with experimental data.

The variation of the coefficients $\alpha_{2}, \alpha_{3}$ and $\alpha_{4}$ with discharge angle $\theta$ was investigated by trying several fitting functions. Figure 5 shows a plot of these coefficients against $\tan (\theta)$ and the respective second degree polynomial fitting lines (forced through the origin as they theoretically should). As the coefficients $\alpha_{2}$ and $\alpha_{4}$ take negative values for all the angles studied, the opposite values are depicted in Figure 5. The analytical expressions for the obtained fitting lines and the corresponding coefficients $R^{2}$ are presented in Table 3. The high values of $R^{2}$ (close to 1 ) show the validity of the proposed fittings, which allow the determination of the polynomial coefficients for any angle in the range studied. 


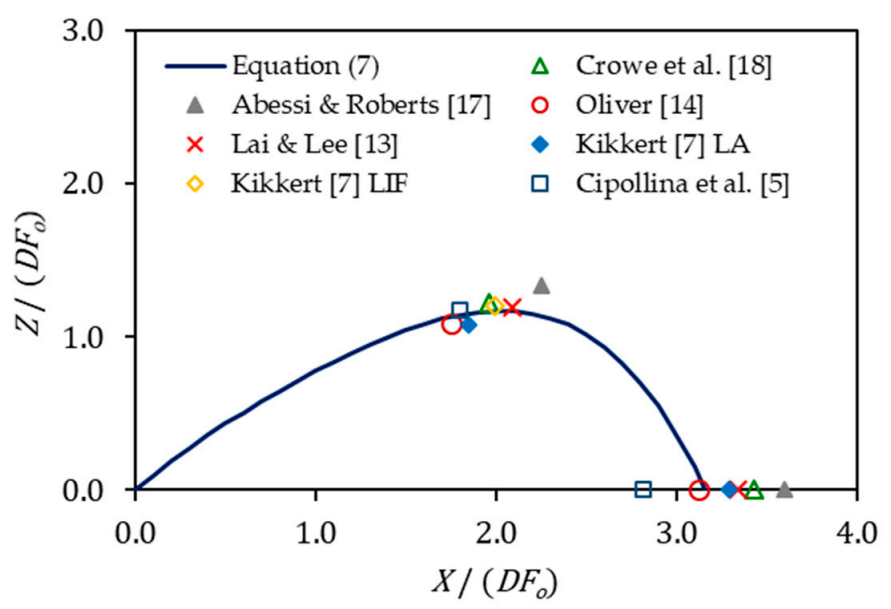

(a)

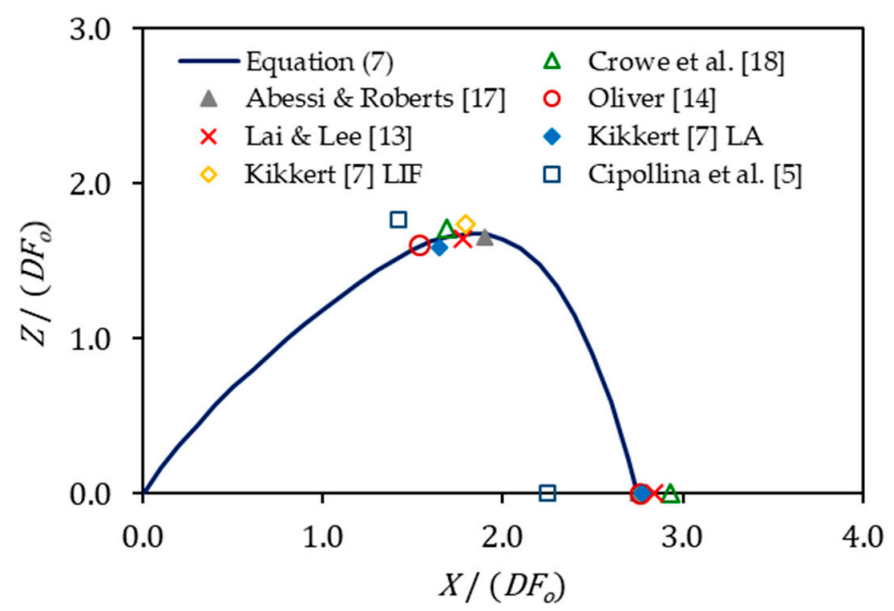

(b)

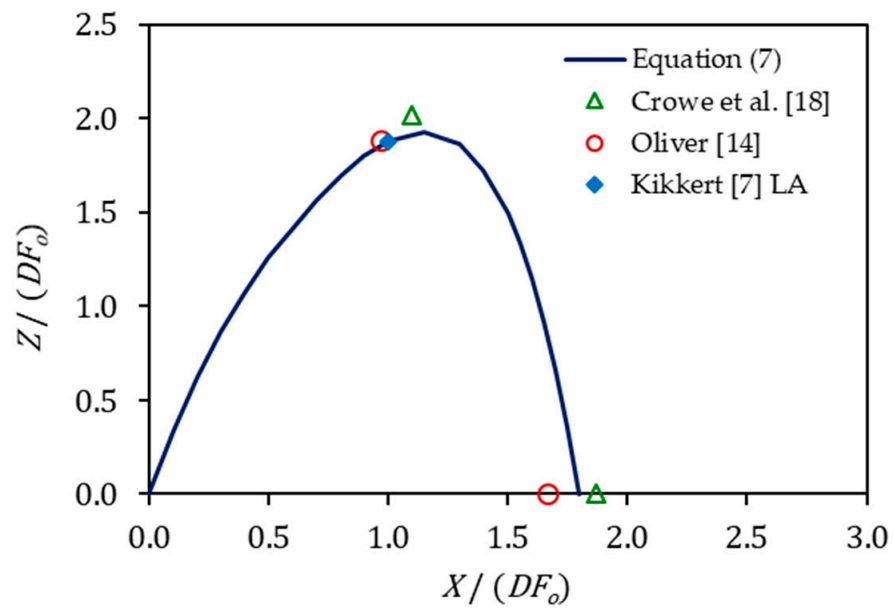

(c)

Figure 4. Comparison of the predicted trajectories for (a) $\theta=45^{\circ}$, (b) $\theta=60^{\circ}$, (c) $\theta=75^{\circ}$ with experimental data for the maximum height and the return point of the jet centerline. 


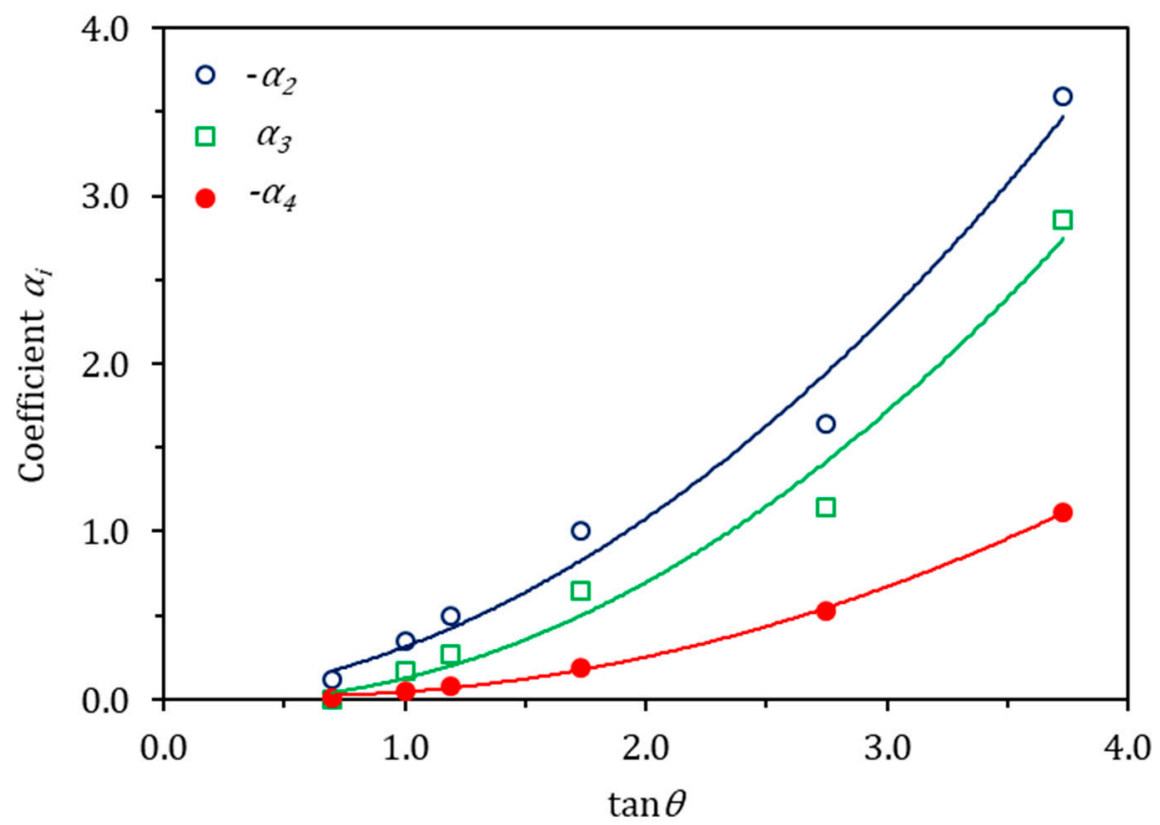

Figure 5. Variation of the coefficients $\alpha_{2}, \alpha_{3}, \alpha_{4}$ of Equation (7) with discharge angle $\theta$ and respective second degree polynomial fittings.

Table 3. Second degree polynomial fitting curves for the variation of coefficients $\alpha_{2}, \alpha_{3}, \alpha_{4}$ with discharge angle $\theta$ and the respective values of $R^{2}$.

\begin{tabular}{ccc}
\hline Coefficient & Polynomial Fitting Curve & $\mathbf{R}^{\mathbf{2}}$ \\
\hline$\alpha_{2}$ & $-\alpha_{2}=0.2259(\tan \theta)^{2}+0.0875 \tan \theta$ & 0.982 \\
$\alpha_{3}$ & $\alpha_{3}=0.223(\tan \theta)^{2}-0.0969 \tan \theta$ & 0.979 \\
$\alpha_{4}$ & $-\alpha_{4}=0.0944(\tan \theta)^{2}-0.0566 \tan \theta$ & 0.999 \\
\hline
\end{tabular}

\subsection{Dilution}

Estimating dilution is particularly important as it can be directly related to the environmental impact of the effluent on the water body. For this reason, several studies have reported experimental results for the minimum dilution at the maximum height $S_{t}$ and the minimum dilution at the return point $S_{i}$, e.g., $\left[12-14,16,17,20\right.$ ]. Zeitoun et al. [2] concluded that (among the angles $30^{\circ}, 45^{\circ}$ and $60^{\circ}$ ) $60^{\circ}$ is optimum, because it results in the longest trajectory and consequently in the highest ultimate dilution. Obviously, the trajectory length affects the dilution, but the variation of the dilution along the trajectory deserves further attention.

Conceivably, an inclined dense jet behaves initially as a simple jet dominated by its initial momentum, whereas buoyancy takes over gradually leading to plume-like behavior around and beyond the maximum height. For simple jets Fischer et al. [33] proposed the following equation:

$$
\frac{C_{m}}{C_{o}}=5.6 \frac{l_{Q}}{z}
$$

where $C_{m}$ is the maximum concentration (at the jet centerline) in excess of the ambient concentration (herein considered zero), $C_{o}$ is the initial (at the source) concentration, $l_{Q}$ is a length scale equal to $\sqrt{\frac{\pi D^{2}}{4}}$ and $z$ is the axial distance from the source.

The previous equation can be rewritten in terms of the centerline dilution as

$$
S=\frac{C_{o}}{C_{m}}=0.20 \frac{z}{D}
$$


For vertical plumes Fischer et al. [33] suggested that

$$
C_{m}=9.1 Q C_{o} B^{-1 / 3} z^{-5 / 3}
$$

where $B$ is the buoyancy flux at the source, defined as $B=g_{o}^{\prime} U_{o} \frac{\pi D^{2}}{4}$. Therefore, Equation (16) can be rewritten as

$$
S=\frac{C_{o}}{C_{m}}=0.129 F_{o}^{-2 / 3}\left(\frac{z}{D}\right)^{5 / 3}
$$

In normalized form, Equations (15) and (17) are as follows:

$$
\begin{gathered}
\frac{S}{F_{o}}=0.20 \frac{z}{D F_{o}} \quad \text { (jets) } \\
\frac{S}{F_{o}}=0.129\left(\frac{z}{D F_{o}}\right)^{5 / 3} \quad \text { (plumes) }
\end{gathered}
$$

showing that for vertical (positively) buoyant jets the dilution follows a $z^{1}$ law in the jet-regime and a $z^{5 / 3}$ law in the plume-regime.

To investigate the relation between dilution and trajectory length in inclined dense jets, the length $L_{1}$ of the dimensionless trajectory from the jet exit up to the maximum centerline height and the total length $L_{\text {tot }}$ of the dimensionless trajectory (up to the return point) were calculated from the fourth degree polynomial (Equation (7)) for each discharge angle, as

$$
\begin{gathered}
L_{1}=\int_{0}^{X_{Z}^{*}} \sqrt{1+\left(\frac{d Z^{*}}{d X^{*}}\right)^{2}} d x \\
L_{t o t}=\int_{0}^{X_{i c}^{*}} \sqrt{1+\left(\frac{d Z^{*}}{d X^{*}}\right)^{2}} d x
\end{gathered}
$$

The calculated lengths of the dimensionless trajectory and the experimental values of normalized dilution reported by Papakonstantis et al. [12] and Papakonstantis and Tsatsara [20] for the respective discharge angles are presented in Table 4 . The maximum values of $L_{1}$ and $L_{\text {tot }}$ were obtained for the angle of $60^{\circ}$, suggesting that the highest dilution at both the maximum height and the return point is expected for a discharge angle of $60^{\circ}$, in accordance with the early observations of Zeitoun et al. [2]. The present experimental results show a slightly higher dilution at the maximum height for $50^{\circ}$ and at the return point for $70^{\circ}$ compared to the respective values for $60^{\circ}$, but the differences are very small. This is consistent with earlier studies indicating that the dilution varies very little in the range of $45^{\circ}$ to $75^{\circ}[6,12]$ or $45^{\circ}$ to $65^{\circ}[16]$.

Table 4. Computed lengths of dimensionless trajectory and experimental values of normalized dilution.

\begin{tabular}{ccccc}
\hline $\boldsymbol{\theta}$ & $\boldsymbol{L}_{1}$ & $\boldsymbol{L}_{\text {tot }}$ & $\boldsymbol{S}_{\boldsymbol{t}} / \boldsymbol{F}_{\boldsymbol{o}}$ & $\boldsymbol{S}_{\boldsymbol{i}} / \boldsymbol{F}_{\boldsymbol{o}}$ \\
\hline $35^{\circ}$ & 2.087 & 3.698 & 0.45 & 1.20 \\
$45^{\circ}$ & 2.384 & 4.114 & 0.52 & 1.55 \\
$50^{\circ}$ & 2.372 & 4.142 & 0.57 & 1.69 \\
$60^{\circ}$ & 2.542 & 4.568 & 0.56 & 1.68 \\
$70^{\circ}$ & 2.249 & 4.283 & 0.47 & 1.76 \\
$75^{\circ}$ & 2.321 & 4.439 & 0.51 & 1.67 \\
\hline
\end{tabular}

In Figure 6, the experimentally obtained normalized dilutions $S / F_{0}$ for the discharge angles $35^{\circ}$, $45^{\circ}, 50^{\circ}, 70^{\circ}, 75^{\circ}$ shown in Table 4 , are plotted against the respective length $L$ of the dimensionless trajectory. More specifically, the values of dilution at the maximum centerline height $\left(S_{t} / F_{o}\right)$ are plotted against $L_{1}$, while the dilution values at the return point $\left(S_{i} / F_{o}\right)$ are plotted against $L_{\text {tot }}$. 
As seen in Figure 6, the data concerning the maximum height may be well fitted by a linear equation (passing through the origin) of the form:

$$
\frac{S}{F_{o}}=0.221 L
$$

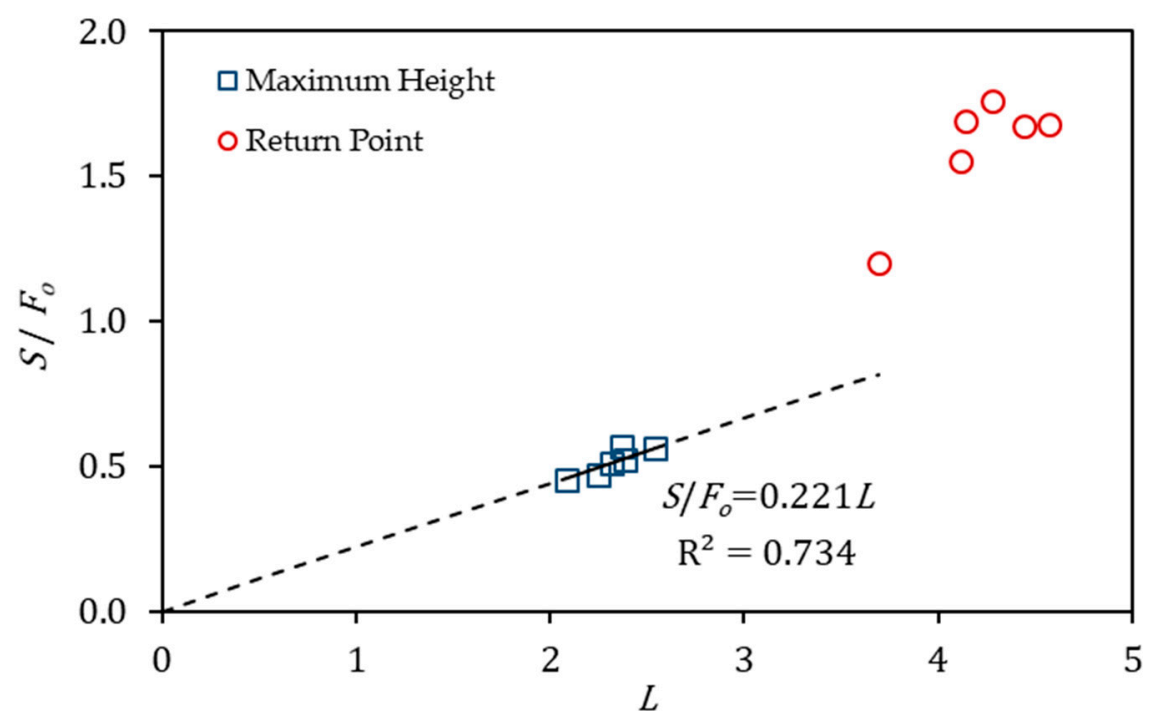

(a)

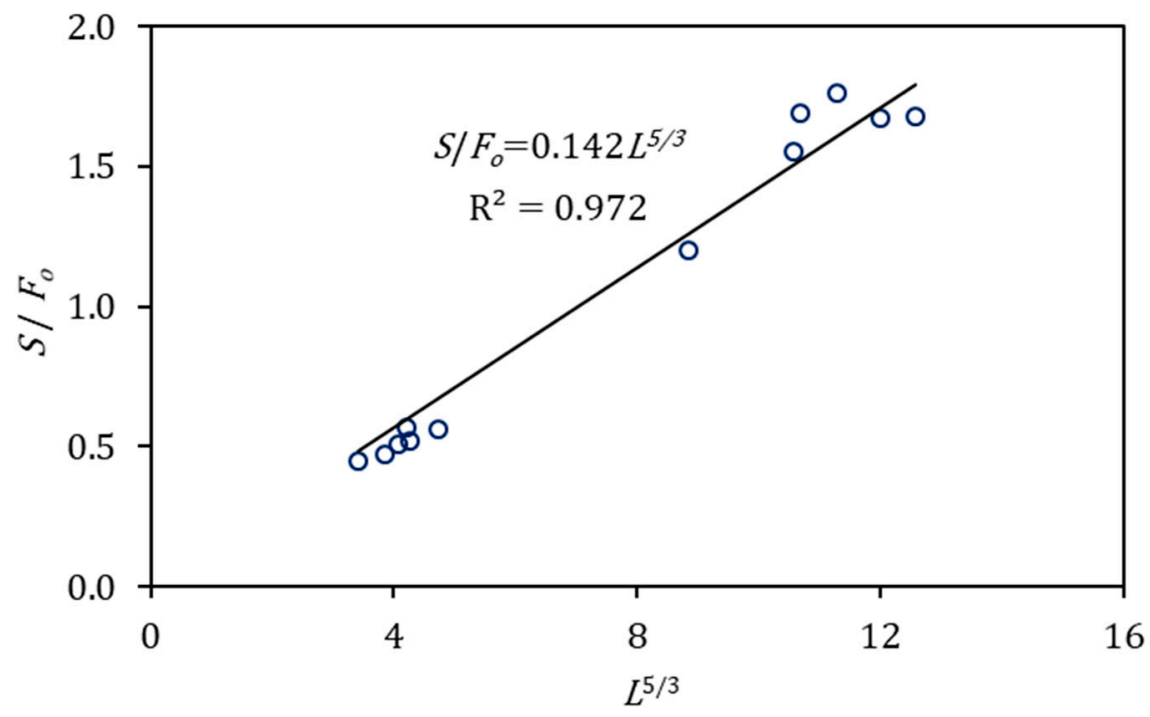

(b)

Figure 6. Normalized dilution against (a) the length $L$ and (b) the length $\mathrm{L}^{5 / 3}$ of the dimensionless trajectory.

This suggests that the upward branch follows the law of the jet-regime with the constant 0.221 being close to 0.20 of Equation (18). However, it is evident that the dilution data at the return point do not follow the same law. As seen in Figure 6b, the entire dilution dataset (i.e., data at both the return point and the maximum height) are well fitted by an equation of the form:

$$
\frac{\mathrm{S}}{F_{0}}=0.142 L^{5 / 3}
$$


Equation (23) implies that the dilution throughout the downward branch of the flow approximately follows the law of the plume-regime, with the constant 0.142 being close to 0.129 of Equation (19).

To further clarify the behavior of the inclined jet in the plume-like regime, the ratio of the normalized dilution $S^{*}\left(S^{*}=S / F_{0}\right)$ to the respective dimensionless length $L^{5 / 3}$ is plotted against the inclination angle $\theta$ in Figure 7. It is evident that (i) the ratios for both the maximum height and the return point are essentially constant over the range of angles studied, and (ii) the values $S_{i}^{*} / L^{5 / 3}$ at the return point are somewhat larger than the values $S_{t}^{*} / L^{5 / 3}$ at the maximum height.

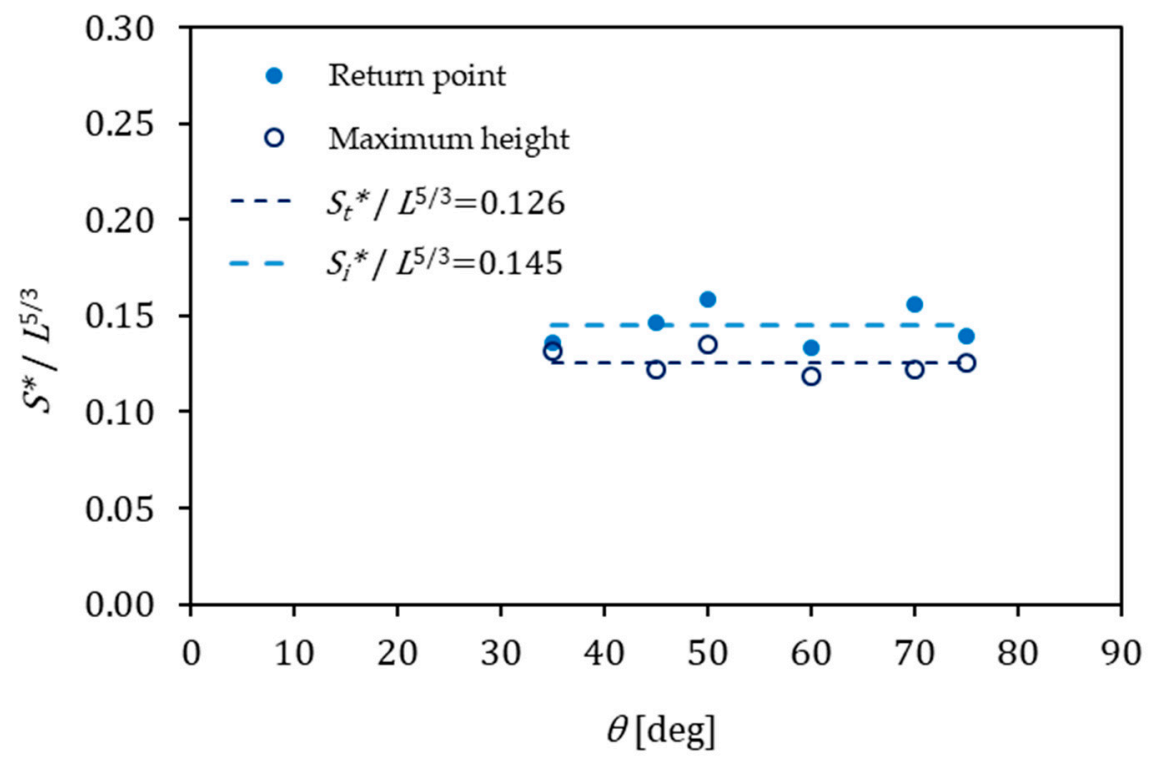

Figure 7. Variation of normalized dilution with inclination angle.

Thus, although the entire dataset follows approximately Equation (23), two subsets can be distinguished considering the average values of the respective ratios:

$$
\begin{gathered}
\frac{S_{t}}{F_{o}}=0.126 L^{5 / 3} \\
\frac{S_{i}}{F_{o}}=0.145 L^{5 / 3}
\end{gathered}
$$

It is remarkable that the constant of Equation (24a) for the maximum height dilution almost coincides with the value of the ordinary plume (Equation (19)); the somewhat higher constant for the return point dilution (Equation (24b)), which is essentially the same as in Equation (23), may be attributed to the loss of fluid mass from the main jet flow occurring beyond the maximum height (e.g., [11]).

Considering the above, one may conclude that the dilution at the maximum height can be estimated well by means of either the law of the jet-like regime (Equation (22)) or the plume-like regime (Equation (24a)). The dilution beyond the maximum height and throughout the downward branch up to the return point follows the law of the plume-like regime (Equation (23)). Since the dense jet is initially momentum-dominated, it is conceivable that Equation (22) will also be valid in the upward branch of the flow. Dilution data at positions before the maximum height could clarify whether the jet-like regime extends up to the maximum height or the flow turns to plume-like behavior before reaching that height.

\section{Conclusions}

A lot of experimental data on inclined dense jets have been obtained in recent years. Knowledge of the location of the maximum height and the return point of the jet centerline for a given inclination 
angle provides five boundary conditions, which allow the determination of a fourth degree polynomial approximation of the jet trajectory in dimensionless form. Available data from past studies of the authors were used in this study for defining the coefficients of this polynomial function for six discharge angles in the range of $35^{\circ}$ to $75^{\circ}$. The resulting trajectories were compared and found to be in satisfactory agreement with experimental results of other investigators. Simple analytical expressions were derived for the dependence of the polynomial coefficients on the inclination angle, therefore the trajectory at any angle in the range studied can be readily determined. Moreover, the proposed model allows for a straightforward computation of the (dimensionless) length of the trajectory at any desired distance from the source. The relation between the computed length and experimentally obtained values of normalized dilution, suggests that the minimum (centerline) dilution of a dense jet follows the linear law of a simple jet in the upward branch and the 5/3 law of a plume in the downward branch. The relevant equations presented allow a simple estimation of the dilution at any point along the trajectory. Overall, the proposed simplified analytical model is expected to be useful for design purposes, providing sufficiently accurate predictions for the geometry and the dilution of inclined dense jets without resorting to experiments or elaborate numerical models.

Author Contributions: Conceptualization, G.C.C.; methodology, G.C.C. and I.G.P.; software, I.G.P.; validation, I.G.P.; formal analysis, I.G.P.; investigation, I.G.P.; data curation, I.G.P.; writing-original draft preparation, G.C.C. and I.G.P.; writing-review and editing, I.G.P. and G.C.C.; visualization, I.G.P.; supervision, G.C.C.; project administration G.C.C. All authors have read and agreed to the published version of the manuscript.

Funding: This research received no external funding.

Conflicts of Interest: The authors declare no conflict of interest.

\section{References}

1. Lattemann, S.; Höpner, T. Environmental impact and impact assessment of seawater desalination. Desalination 2008, 220, 1-15. [CrossRef]

2. Zeitoun, M.A.; Mcllhenny, W.F.; Reid, R.O. Conceptual Designs of Outfall Systems for Desalting Plants; Res. and Devel. Progress Report No 550; Office of Saline Water, US Dept of Interior: Washington, DC, USA, 1970.

3. Roberts, P.J.W.; Toms, G. Inclined dense jets in flowing current. J. Hydraul. Eng. 1987, 113, 323-341. [CrossRef]

4. Roberts, P.J.W.; Ferrier, A.; Daviero, G. Mixing in inclined dense jets. J. Hydraul. Eng. 1997, 123, 693-699. [CrossRef]

5. Cipollina, A.; Brucato, A.; Grisafi, F.; Nicosia, S. Bench-scale investigation of inclined dense jets. J. Hydraul. Eng. 2005, 131, 1017-1022. [CrossRef]

6. Nemlioglu, S.; Roberts, P.J.W. Experiments on Dense Jets Using Three-Dimensional Laser-Induced Fluorescence (3DLIF). In Proceedings of the 4th International Conference on Marine Waste Water Disposal and Marine Environment \& 2nd International Exhibition on Materials Equipment and Services for Coastal WWTP, Outfalls and Sealines, Antalya, Turkey, 6-10 November 2006.

7. Kikkert, G.A. Buoyant Jets with Two and Three-Dimensional Trajectories. Ph.D. Thesis, University of Canterbury, Christchurch, New Zealand, 2006.

8. Kikkert, G.A.; Davidson, M.J.; Nokes, R.I. Inclined negatively buoyant discharges. J. Hydraul. Eng. 2007, 133, 545-554. [CrossRef]

9. Ferrari, S.; Querzoli, G. Mixing and re-entrainment in a negatively buoyant jet. J. Hydraul. Res. 2010, 48, 632-640. [CrossRef]

10. Shao, D.; Law, A.W.K. Mixing and boundary interactions of $30^{\circ}$ and $45^{\circ}$ inclined dense jets. Environ. Fluid Mech. 2010, 10, 521-553. [CrossRef]

11. Papakonstantis, I.G.; Christodoulou, G.C.; Papanicolaou, P.N. Inclined negatively buoyant jets 1: Geometrical characteristics. J. Hydraul. Res. 2011, 49, 3-12. [CrossRef]

12. Papakonstantis, I.G.; Christodoulou, G.C.; Papanicolaou, P.N. Inclined negatively buoyant jets 2: Concentration measurements. J. Hydraul. Res. 2011, 49, 13-22. [CrossRef]

13. Lai, C.C.K.; Lee, J.H.W. Mixing of inclined dense jets in stationary ambient. J. Hydro Environ. Res. 2012, 6, 9-28. [CrossRef]

14. Oliver, C.J. Near Field Mixing of Negatively Buoyant Jets. Ph.D. Thesis, University of Canterbury, Christchurch, New Zealand, 2012. 
15. Oliver, C.J.; Davidson, M.J.; Nokes, R.I. Removing the boundary influence on negatively buoyant jets. Environ. Fluid Mech. 2013, 13, 625-648. [CrossRef]

16. Abessi, O.; Roberts, P.J.W. Effect of nozzle orientation on dense jets in stagnant environments. J. Hydraul. Eng. 2015, 141, 06015009. [CrossRef]

17. Abessi, O.; Roberts, P.J.W. Dense jet discharges in shallow water. J. Hydraul. Eng. 2016, 142, 04015033. [CrossRef]

18. Crowe, A.T.; Davidson, M.J.; Nokes, R.I. Velocity measurements in inclined negatively buoyant jets. Environ. Fluid Mech. 2016, 16, 503-520. [CrossRef]

19. Papakonstantis, I.G.; Tsatsara, E.I. Trajectory Characteristics of Inclined Turbulent Dense Jets. Environ. Process. 2018, 5, 539-554. [CrossRef]

20. Papakonstantis, I.G.; Tsatsara, E.I. Mixing Characteristics of Inclined Turbulent Dense Jets. Environ. Process. 2019, 6, 525-541. [CrossRef]

21. Vafeiadou, P.; Papakonstantis, I.; Christodoulou, G. Numerical simulation of inclined negatively buoyant jets. In Proceedings of the 9th International Conference on Environmental Science and Technology, Rhodes, Greece, 1-3 September 2005; pp. A1537-A1542.

22. Oliver, C.J.; Davidson, M.J.; Nokes, R.I. k- $\varepsilon$ Predictions of the initial mixing of desalination discharges. Environ. Fluid Mech. 2008, 8, 617-625. [CrossRef]

23. Kheirkhah Gildeh, H.; Mohammadian, A.; Nistor, I.; Qiblawey, H. Numerical modeling of $30^{\circ}$ and $45^{\circ}$ inclined dense turbulent jets in stationary ambient. Environ. Fluid Mech. 2015, 15, 537-562. [CrossRef]

24. Kheirkhah Gildeh, H.; Mohammadian, A.; Nistor, I.; Qiblawey, H.; Yan, X. CFD modeling and analysis of the behavior of $30^{\circ}$ and $45^{\circ}$ inclined dense jets-New numerical insights. J. Appl. Water Eng. Res. 2016, 4, 112-127. [CrossRef]

25. Zhang, S.; Jiang, B.; Law, A.W.K.; Zhao, B. Large eddy simulations of $45^{\circ}$ inclined dense jets. Environ. Fluid Mech. 2016, 16, 101-121. [CrossRef]

26. Zhang, S.; Law, A.W.K.; Jiang, M. Large eddy simulations of $45^{\circ}$ and $60^{\circ}$ inclined dense jets with bottom impact. J. Hydro Environ. Res. 2017, 15, 54-66. [CrossRef]

27. Papanicolaou, P.N.; Papakonstantis, I.G.; Christodoulou, G.C. On the entrainment coefficient in negatively buoyant jets. J. Fluid Mech. 2008, 614, 447-470. [CrossRef]

28. Yannopoulos, P.C.; Bloutsos, A.A. Escaping mass approach for inclined plane and round buoyant jets. J. Fluid Mech. 2012, 695, 81-111. [CrossRef]

29. Oliver, C.J.; Davidson, M.J.; Nokes, R.I. Predicting the near-field mixing of desalination discharges in a stationary environment. Desalination 2013, 309, 148-155. [CrossRef]

30. Palomar, P.; Lara, J.L.; Losada, I.J. Near field brine discharge modeling part 2: Validation of commercial tools. Desalination 2012, 290, 28-42. [CrossRef]

31. Nikiforakis, I.K.; Stamou, A.I.; Christodoulou, G.C. A modified integral model for negatively buoyant jets in a stationary ambient. Environ. Fluid Mech. 2015, 15, 939-957. [CrossRef]

32. Christodoulou, G.C.; Papakonstantis, I.G. Simplified estimates of trajectory of inclined negatively buoyant jets. In Proceedings of the Environmental Hydraulics_Proceedings of the 6th International Symposium on Environmental Hydraulics, Athens, Greece, 23-25 June 2010; Christodoulou, G.C., Stamou, A.I., Eds.; Taylor \& Francis Group: London, UK, 2010; Volume 1, pp. 165-170.

33. Fischer, H.B.; List, E.J.; Koh, R.C.Y.; Imberger, J.; Brooks, N.H. Mixing in Inland and Coastal Waters; Academic Press: San Diego, CA, USA, 1979.

Publisher's Note: MDPI stays neutral with regard to jurisdictional claims in published maps and institutional affiliations.

(C) 2020 by the authors. Licensee MDPI, Basel, Switzerland. This article is an open access article distributed under the terms and conditions of the Creative Commons Attribution (CC BY) license (http://creativecommons.org/licenses/by/4.0/). 